\title{
Détection et localisation d'endommagements structuraux par la méthode des sous-espaces, le modèle de Kalman et la méthode d'analyse en composantes principales
}

\author{
Ai-Min Yan ${ }^{\mathrm{a}}$, Pascal De Boe et Jean-Claude Golinval \\ Département AéroSpatiale Mécanique (ASMA), LTAS - Vibrations et Identification des Structures, \\ Université de Liège, 1 chemin des chevreuils, Bât. B52, 4000 Liège, Belgique
}

Reçu le 29 juillet 2004, accepté le 2 mars 2006

\begin{abstract}
Résumé - Cet article porte sur l'utilisation de techniques d'analyse de processus statistiques pour la détection et la localisation d'endommagements structuraux à partir de mesures vibratoires. La première approche proposée dans ce travail se base sur la méthode d'identification des sous-espaces stochastiques pour construire un modèle de Kalman représentatif de l'état initial (de référence) de la structure. Ce modèle est alors utilisé pour réaliser une prédiction des réponses nouvellement mesurées. L'analyse statistique de l'erreur de reconstruction du modèle permet de définir un critère de détection de l'apparition d'un défaut. L'intérêt de cette méthode est que seule l'identification du modèle pour les données de référence est nécessaire. La détection de l'endommagement peut alors être effectuée de manière automatique par surveillance de la structure sans nécessiter de nouvelle identification. Dans la seconde approche, l'analyse en composantes principales des réponses est utilisée pour extraire les directions principales (les caractéristiques) permettant de définir un sous-espace représentatif du comportement dynamique de la structure. Le moindre changement dans la réponse d'un capteur affecte l'espace sous-tendu par l'ensemble de tous les capteurs. Par conséquent, la comparaison entre les sous-espaces correspondant respectivement à la structure saine et la structure actuelle (potentiellement endommagée) permet de détecter l'apparition éventuelle d'un endommagement. L'analyse en composantes principales peut également être réalisée sur un sous-ensemble de capteurs dans le but de localiser le(s) capteur(s) responsable(s) de l'apparition du défaut, et par conséquent, la sous-structure endommagée.
\end{abstract}

Mots clés : Diagnostic d'endommagement structural / détection de défauts / modèle de Kalman / méthode d'identification des sous-espaces stochastiques / analyse en composantes principales

\begin{abstract}
Structural damage diagnosis based on stochastic subspace identification, Kalman model, and principal component analysis. This paper deals with the application of statistical process control techniques for damage diagnosis based on vibration measurements. The first approach considered in this work is based the Stochastic Subspace Identification (SSI) algorithm, from which a Kalman model is constructed to fit the measured response histories of the undamaged (reference) structure. This model may be used to make a prediction of the newly measured responses. The residual error between the model predictions and the actual measurements is defined as a damage-sensitive feature. Outlier statistics provides then a quantitative indicator of damage. The advantage of the method is that model extraction has to be performed only once using the reference data and that no further modal identification is needed. Thus on-line structural health monitoring may easily be realized. In the second approach, principal component analysis (PCA) of the sensor time-responses is used to extract principal directions (i.e. features), which define a subspace that is representative of the dynamics of the instrumented structure. Any change in the response of a single sensor affects the subspace spanned by the complete sensor response set. It follows that the subspace corresponding to the current state of the structure can be compared to the subspace of the initial state of the structure, assumed to be healthy, in order to diagnose possible damage. Principal component analysis may also be performed for every potential subset of damaged sensors in order to identify the involved sensor, and, therefore, the damaged substructure.
\end{abstract}

Key words: Structural health monitoring / damage detection / Kalman model / stochastic subspace identification / principal component analysis

a Auteur correspondant : am.yan@ulg.ac.be 


\section{Introduction}

La détection d'endommagements à partir de mesures vibratoires nécessite l'extraction de paramètres caractéristiques de la structure à partir d'échantillons temporels périodiquement espacés, ainsi que l'analyse de ces paramètres caractéristiques afin de déterminer à chaque instant l'état d'intégrité du système. Si l'on dispose d'un modèle par éléments finis (EF) de la structure, la détection et la localisation du dommage peuvent être réalisées à partir de méthodes de correction paramétrique de modèles (recalage) [1]. Une autre méthode consiste à identifier toutes modifications des paramètres modaux (fréquences naturelles, modes propres et amortissement) [2]. Dans ce travail, on considère tout d'abord une technique d'identification basée sur la méthode stochastique des sous-espaces (SSI pour Stochastic Subspace Identification) [3]. Cette méthode a déjà été appliquée avec succès dans le cas de structures de génie civil [4]. Cependant, le diagnostic de défauts peut également être réalisé sans recourir à la construction d'un modèle EF ni à une technique d'identification des paramètres modaux $[5,6]$. Dans ce travail, deux méthodes « stochastiques » basées sur une analyse statistique sont proposées. La première approche [7] est liée à la méthode SSI, à partir de laquelle un modèle de Kalman est extrait des réponses temporelles mesurées sur la structure saine. Les données successives sont alors exploitées pour détecter si les paramètres caractéristiques du système s'écartent de manière significative ou non de la norme de référence. Une idée similaire est adoptée pour la seconde approche basée sur la décomposition en composantes principales [8].

\section{Identification modale et détection de défauts par une méthode de sous-espaces}

L'équation d'équilibre dynamique d'une structure linéaire à plusieurs degrés de liberté s'écrit sous la forme générale :

$$
\mathbf{M} \ddot{z}(t)+\mathbf{D} \dot{z}(t)+\mathbf{K} \boldsymbol{z}(t)=\boldsymbol{f}(t)
$$

où $\mathbf{M}, \mathbf{D}$ et $\mathbf{K}$ désignent respectivement les matrices de masse, d'amortissement et de raideur du système; $\boldsymbol{f}(t)$ représente le vecteur des forces d'excitation et $\boldsymbol{z}(t)$ le vecteur des degrés de liberté. Dans l'espace d'état, l'équation (1) prend la forme :

$$
\dot{\boldsymbol{x}}(t)=\mathbf{A}_{\mathrm{c}} \boldsymbol{x}(t)+\mathbf{B}_{\mathrm{c}} \boldsymbol{w}(t)
$$

avec les définitions suivantes :

$$
\begin{aligned}
\boldsymbol{x}(t) & =\left\{\begin{array}{l}
\dot{\boldsymbol{z}}(t) \\
\boldsymbol{z}(t)
\end{array}\right\}, \quad \boldsymbol{w}(t)=\left\{\begin{array}{l}
\boldsymbol{f}(t) \\
\mathbf{0}
\end{array}\right\} \\
\mathbf{A}_{\mathrm{c}} & =\left[\begin{array}{cc}
-\mathbf{M}^{-1} \mathbf{D} & -\mathbf{M}^{-1} \mathbf{K} \\
\mathbf{I} & \mathbf{0}
\end{array}\right], \quad \mathbf{B}_{\mathrm{c}}=\left[\begin{array}{cc}
\mathbf{M}^{-1} & \mathbf{0} \\
\mathbf{0} & \mathbf{0}
\end{array}\right]
\end{aligned}
$$

En posant $t_{k}=k \Delta t$ (où $\Delta t$ désigne la période d'échantillonnage), on obtient la représentation du modèle d'état sous forme discrète, à savoir :

$$
\begin{aligned}
\boldsymbol{x}_{k+1} & =\mathbf{A} \boldsymbol{x}_{k}+\boldsymbol{w}_{k} \\
\boldsymbol{y}_{k} & =\mathbf{C} \boldsymbol{x}_{k}+\boldsymbol{v}_{k}
\end{aligned}
$$

où $\mathbf{A} \in \Re^{n \times n}$ et $\mathbf{C} \in \Re^{\ell \times n}$ sont appelées respectivement matrice d'état et matrice des observations du système; $\boldsymbol{x}_{k}$ est le vecteur des variables d'état de dimension $n$ (l'ordre du système à déterminer) et $\boldsymbol{y}_{k}$ est le vecteur des observations de dimension $\ell$ (le nombre de capteurs). $\boldsymbol{w}_{k}$ et $\boldsymbol{v}_{k}$ sont des vecteurs de variables d'entrée liées respectivement au bruit associé au processus et au bruit de mesures. On notera que l'excitation, inconnue a priori, est prise en compte implicitement dans les termes liés au bruit. Le bruit est supposé être un bruit blanc gaussien de moyenne nulle.

La méthode SSI (Stochastic Subspace Identification) consiste à identifier les matrices $(\mathbf{A}, \mathbf{C})$ à partir de la seule connaissance du vecteur des variables de sortie $\boldsymbol{y}_{k}$ (mesures effectuées aux $\ell$ capteurs en termes de déplacements, de vitesses ou d'accélérations). Dans la suite, deux variantes de la méthode SSI sont considérées.

\subsection{Identification basée sur les covariances des observations (covariance-driven SSI)}

Soit $\mathbf{H}_{p, q}$ la matrice de Hankel définie à partir des matrices de covariance $\Lambda_{i}$ de la manière suivante $(p \leq q)$ :

$$
\mathbf{H}_{p, q}=\left[\begin{array}{ccccc}
\boldsymbol{\Lambda}_{1} & \boldsymbol{\Lambda}_{2} & \ldots & \ldots & \boldsymbol{\Lambda}_{q} \\
\boldsymbol{\Lambda}_{2} & \boldsymbol{\Lambda}_{3} & \ldots & \ldots & \boldsymbol{\Lambda}_{q+1} \\
\ldots & \ldots & \ldots & \ldots & \ldots \\
\boldsymbol{\Lambda}_{p} & \boldsymbol{\Lambda}_{p+1} & \ldots & \ldots & \boldsymbol{\Lambda}_{p+q-1}
\end{array}\right]
$$

Remarquons que la matrice $\boldsymbol{\Lambda}_{i}$ peut être approchée par : $\boldsymbol{\Lambda}_{i} \approx \frac{1}{N-i} \sum_{k=1}^{N-i} \boldsymbol{y}_{k+i} \boldsymbol{y}_{k}^{T}$ où $N$ correspond au nombre d'échantillons pris en compte dans l'approximation.

On sait par la théorie des sous-espaces [3] que la matrice de Hankel peut être écrite sous la forme du produit de la matrice d'observabilité $\boldsymbol{O}_{p}$ (d'ordre $p$ ) et de la matrice de contrôlabilité $\boldsymbol{C}_{q}$ (d'ordre $q$ et de rang $n$ correspondant à l'ordre du système) :

$$
\mathbf{H}_{p, q}=\mathbf{O}_{p} \mathbf{C}_{q}
$$

avec les définitions

$$
\begin{aligned}
\mathbf{O}_{p} & =\left[\begin{array}{llll}
\mathbf{C} & \mathbf{C A} & \ldots & \mathbf{C A}^{p-1}
\end{array}\right]^{\mathrm{T}} \\
\mathbf{C}_{q} & =\left[\begin{array}{llll}
\mathbf{G} & \mathbf{A G} & \ldots & \mathbf{A}^{q-1} \mathbf{G}
\end{array}\right]
\end{aligned}
$$

et $\mathbf{G}=\mathbf{E}\left[\boldsymbol{x}_{k+1} \boldsymbol{y}_{k}^{\mathrm{T}}\right]$, la matrice de covariance entre les variables d'état et de sortie. 
En pratique, la factorisation de la matrice de Hankel peut être réalisée par décomposition en valeurs singulières, soit :

$$
\mathbf{H}_{p, q}=\left[\mathbf{U}_{1} \mathbf{U}_{2}\right]\left[\begin{array}{cc}
\mathbf{S}_{1} & \mathbf{0} \\
\mathbf{0} & \mathbf{S}_{2}
\end{array}\right]\left[\mathbf{V}_{1} \mathbf{V}_{2}\right]^{\mathrm{T}} \approx \mathbf{U}_{1} \mathbf{S}_{1} \mathbf{V}_{1}^{\mathrm{T}}
$$

où l'on suppose que les valeurs singulières associées à la sous-matrice $\mathbf{S}_{2}$ sont négligeables car elles correspondent aux bruits de mesure. Sous cette condition, l'ordre du système $n$ est déterminé par la dimension de $\mathbf{S}_{1}$. Par comparaison entre les équations (6) et (7), on peut exprimer que :

$$
\begin{aligned}
\mathbf{O}_{p} & =\mathbf{U}_{1} \mathbf{S}_{1}^{1 / 2} \\
\mathbf{C}_{q} & =\mathbf{S}_{1}^{1 / 2} \mathbf{V}_{1}^{\mathrm{T}}
\end{aligned}
$$

Une fois les matrices $\mathbf{O}_{p}$ et $\mathbf{C}_{q}$ identifiées, l'estimation de la matrice d'état $\mathbf{A}$ et de la matrice des observations $\mathbf{C}$ est directe. Par exemple, on voit que la matrice $\mathbf{C}$ correspond aux $\ell$ premières lignes de la matrice des observations $\mathbf{O}_{p}$. De manière équivalente, il est possible d'identifier les matrices $\mathbf{G}$ et $\mathbf{A}$ à partir de la matrice de contrôlabilité $\mathbf{C}_{q}$.

\subsection{Identification basée sur les données (data-driven SSI)}

Dans cette variante de la méthode, la matrice de Hankel est directement formée à partir des réponses mesurées :

$$
\begin{array}{r}
\mathbf{H}_{1,2 i}=\frac{1}{\sqrt{j}}\left[\begin{array}{cccc}
\boldsymbol{y}_{1} & \boldsymbol{y}_{2} & \cdots & \boldsymbol{y}_{j} \\
\cdots & \cdots & \cdots & \cdots \\
\boldsymbol{y}_{i} & \boldsymbol{y}_{i+1} & \cdots & \boldsymbol{y}_{i+j-1} \\
\hline \boldsymbol{y}_{i+1} & \boldsymbol{y}_{i+2} & \cdots & \boldsymbol{y}_{i+j} \\
\cdots & \cdots & \cdots & \cdots \\
\boldsymbol{y}_{2 i} & \boldsymbol{y}_{2 i+1} & \cdots & \boldsymbol{y}_{2 i+j-1}
\end{array}\right] \\
\equiv\left(\frac{\mathbf{H}_{1, i}}{\mathbf{H}_{i+1,2 i}}\right) \equiv\left(\frac{\mathbf{Y}_{p}}{\mathbf{Y}_{f}}\right) \frac{\text { « passé » }}{\text { «utur } »}
\end{array}
$$

où $j=N-2 i+1$. Elle est scindée en deux parties : un bloc de $i$ lignes correspondant aux instants « passés » et un bloc de $i$ lignes correspondant aux instants « futurs». Le processus d'identification est ensuite assez similaire au précédent. Les détails de la méthode peuvent être trouvés dans la référence [3]. La particularité de cette variante de la méthode des sous-espaces est qu'elle permet d'identifier un observateur de Kalman utile pour la détection de défauts [7].

\subsection{Identification des paramètres modaux}

Dès que la matrice d'état du système a été déterminée, les paramètres modaux de la structure peuvent être identifiés en résolvant le problème aux valeurs propres :

$$
\mathrm{A} \Psi=\Psi \Lambda
$$

La matrice diagonale $\boldsymbol{\Lambda}$ contient les valeurs propres $\tau_{i}$, à partir desquelles les fréquences naturelles $f_{i}$ et les facteurs d'amortissement $\zeta_{i}$ du système peuvent être calculés :

$$
\left\{\begin{array}{c}
f_{i}=\frac{\omega_{i}}{2 \pi}=\frac{\left|\ln \left(\tau_{i}\right)\right|}{2 \pi \Delta t} \\
\zeta_{i}=-\frac{\operatorname{Real}\left(\ln \left(\tau_{i}\right)\right)}{\left|\ln \left(\tau_{i}\right)\right|}
\end{array}\right.
$$

$\omega_{i}$ étant la pulsation propre $\mathrm{n}^{\circ} i$.

La matrice modale $\boldsymbol{\Phi}$ (de dimension égale au nombre de degrés de liberté mesurés) peut être obtenue à partir de la matrice des vecteurs propres $\boldsymbol{\Psi}$ (qui sont ici exprimés dans l'espace d'état) à partir de la relation :

$$
\Phi=\mathbf{C} \Psi
$$

\subsection{Détection et localisation de défauts}

La détection de défauts s'effectue par comparaison des paramètres modaux correspondant respectivement à la structure saine (état de référence) et à la structure endommagée (état actuel). Il est possible d'évaluer de manière probabiliste le degré d'incertitude sur les paramètres modaux identifiés.

La localisation du dommage quant à elle peut être basée sur une analyse combinée du changement de raideur et de flexibilité de la structure, lesquelles sont estimées à partir des paramètres modaux identifiés [9].

\section{Diagnostic d'endommagement par le modèle de Kalman}

On sait par la théorie du contrôle que la prédiction des réponses d'un système soumis à une excitation stochastique peut s'effectuer à l'aide d'un modèle de Kalman. Désignons par $\hat{\boldsymbol{x}}_{k+1}$ la prédiction optimale du vecteur d'état $\boldsymbol{x}_{k+1}$ basée sur les matrices d'état du système (3)-(4) et sur les vecteurs de sortie disponibles jusqu'à l'instant $t_{k}$. On a :

$$
\hat{\boldsymbol{y}}_{k}=\mathbf{C} \hat{\boldsymbol{x}}_{k}
$$

Dans le cas d'un système linéaire invariant, les deux prédicteurs sont liés par les relations :

$$
\begin{gathered}
\hat{\boldsymbol{x}}_{k+1}=\mathbf{A} \hat{\boldsymbol{x}}_{k}+\mathbf{K}_{k} \boldsymbol{e}_{k} \\
\boldsymbol{y}_{k}=\mathbf{C} \hat{\boldsymbol{x}}_{k}+\boldsymbol{e}_{k}
\end{gathered}
$$

où $\mathbf{K}_{k} \in \Re^{n \times \ell}$ est la matrice de gain de Kalman et $\boldsymbol{e}_{k}$ le vecteur des erreurs de reconstruction (bruit blanc gaussien de moyenne nulle). Au démarrage, le filtre de Kalman (14)-(15) est le siège d'une phase transitoire pendant laquelle le prédicteur d'état n'est pas stationnaire. Cependant, si la matrice d'état $\mathbf{A}$ est stable, le filtre atteint rapidement un état stationnaire et la matrice de covariance du vecteur d'état $\hat{\boldsymbol{x}}_{k}$ reste constante, ce qui implique que le gain de Kalman est également constant, 
i.e. $\mathbf{K}_{k}=\mathbf{K}$. Le filtre de Kalman travaille alors en état stationnaire.

L'application de l'observateur de Kalman pour le diagnostic de l'endommagement structural est décrit dans [7]. Partant de l'état « initial $» \boldsymbol{x}_{0}=\mathbf{0}$ et $\boldsymbol{e}_{0}=\mathbf{0}$, le vecteur d'état à l'étape $\mathrm{n}^{\circ} k$ et l'erreur de reconstruction correspondante sont calculés par :

$$
\begin{gathered}
\hat{\boldsymbol{x}}_{k}=\mathbf{A} \hat{\boldsymbol{x}}_{k-1}+\mathbf{K} \boldsymbol{e}_{k-1} \\
\boldsymbol{e}_{k}=\boldsymbol{y}_{k}-\hat{\boldsymbol{y}}_{k}=\boldsymbol{y}_{k}-\mathbf{C} \hat{\boldsymbol{x}}_{k}
\end{gathered}
$$

Une séquence d'erreurs de reconstruction peut alors être obtenue par un processus itératif. Cette séquence d'erreurs peut être considérée comme une caractéristique sensible à l'état d'endommagement de la structure si on fait l'hypothèse que le modèle de Kalman de la structure saine n'est plus capable de reproduire les réponses nouvellement mesurées lorsqu'un endommagement apparaît.

L'erreur de reconstruction peut être définie, au cours du temps, au moyen d'un indicateur appelé Novelty In$\operatorname{dex}(N I)$. Celui-ci peut être défini par sa norme euclidienne [5] :

$$
N I_{k}^{\mathrm{E}}=\left\|\boldsymbol{e}_{k}\right\|
$$

ou par sa norme de Mahalanobis :

$$
N I_{k}^{\mathrm{M}}=\sqrt{\boldsymbol{e}_{k}^{\mathrm{T}} \Sigma^{-1} \boldsymbol{e}_{k}}
$$

avec $\Sigma=\frac{1}{N} \mathbf{Y} \mathbf{Y}^{\mathrm{T}}$, la matrice de covariance des observations, $N$ le nombre d'observations et $\mathbf{Y}$ la matrice des observations définie par la relation (20).

En l'absence d'endommagement, le niveau de l'erreur de reconstruction reste faible. Conformément à la théorie de l'identification par la méthode des sous-espaces, les erreurs de reconstruction des réponses par le modèle de Kalman répondent à une distribution normale gaussienne, ainsi que les indicateurs (18) et (19). Pour cette raison, on peut se baser sur une analyse statistique pour donner une mesure quantitative de l'apparition du niveau d'endommagement [7]. Par exemple, on peut compter le nombre de fois que l'indicateur NI dépasse un seuil de référence correspondant au triple de la déviation standard des indices NI correspondant à la structure saine.

\section{Détection d'endommagement par l'analyse en composantes principales}

L'analyse en composantes principales (en anglais «Principal component analysis » ou PCA) permet d'étudier des données dans un espace de dimensions réduites en remplaçant les données corrélées par de nouvelles variables : les composantes principales. Bien que la méthode de l'analyse en composantes principales ait été considérée depuis longtemps dans le domaine $\mathrm{du}$ traitement des images et pour le traitement statistique de données provenant d'expérimentations chimiques et biologiques, ce n'est que récemment que cet outil mathématique a trouvé des applications concrètes dans le domaine de la dynamique des structures $[6,8,10]$.

Considérons la matrice des observations $\mathbf{Y}$ construite à partir des réponses en $\ell$ capteurs et en $N$ instants $(N \gg$ $\ell)$, à savoir :

$$
\mathbf{Y}=\left[\begin{array}{ccc}
y_{11} & \cdots & y_{1 N} \\
\vdots & \ddots & \vdots \\
y_{\ell 1} & \cdots & y_{\ell N}
\end{array}\right]
$$

La décomposition en valeurs singulières (SVD) constitue un moyen efficace de détermination des caractéristiques principales (les valeurs et vecteurs propres) de la matrice des observations $\mathbf{Y}$ permettant d'écrire l'égalité suivante :

$$
\mathbf{Y} \approx \mathbf{U}_{1} \mathbf{S}_{1} \mathbf{V}_{1}^{T}
$$

où $\mathbf{U}$ est une matrice orthonormée de dimension $(\ell \times m)$, $(m<\ell)$; l'ensemble des $m$ colonnes forme l'hyperplan des composantes principales, dans lequel reposent les différentes observations. Chaque colonne de $\mathbf{U}$ est associée aux $N$ coefficients de la matrice $\mathbf{V}$, représentant, au cours du temps, les amplitudes des différentes composantes principales. La diagonale principale de la matrice $\mathbf{S}_{1}$, de dimension $(\ell \times m)$, donne les valeurs singulières, triées par ordre décroissant, de la matrice $\mathbf{Y}$. Les valeurs singulières sont représentatives de l'importance de chaque composante principale. Dans le cas structural, ceci signifie que les mouvements d'une structure seront préférentiellement orientés suivant les directions principales associées aux plus grandes valeurs singulières.

L'expression (21) montre que chaque vecteur de réponses peut également être écrit sous la forme d'une combinaison linéaire de vecteurs :

$$
\boldsymbol{y}(t)=\sum_{i=1}^{m} \beta_{i}(t) \mathbf{U}^{i}
$$

D'une manière générale, on sait par la théorie des vibrations que la réponse dynamique d'une structure peut toujours s'écrire sous la forme :

$$
\boldsymbol{y}(t)=\sum_{i=1}^{m} \alpha_{i}(t) \boldsymbol{\Phi}^{i}+\alpha_{r}(t) \boldsymbol{R}
$$

où $\boldsymbol{y}$ désigne le vecteur des réponses mesurées aux $\ell$ capteurs; $\Phi^{i}$ est le mode propre $\mathrm{n}^{\circ} i ; m$ est le nombre de modes propres considérés dans la bande de fréquences d'analyse; $\boldsymbol{R}$ représente la contribution quasi-statique des modes résiduels et $\alpha_{i}(t), \alpha_{r}(t)$ sont les coordonnées modales.

Les relations (22) et (23) montrent que l'espace (ou hyperplan) couvert par les composantes principales est équivalent à l'hyperplan généré par les $m$ premiers modes propres structuraux et par le vecteur $\boldsymbol{R}$ décrivant l'ensemble des modes résiduels, si le nombre de capteurs est suffisamment important.

La figure 1a donne l'exemple d'une structure quelconque, instrumentée par trois capteurs de déplacement, et réagissant suivant ses deux premiers modes propres. 

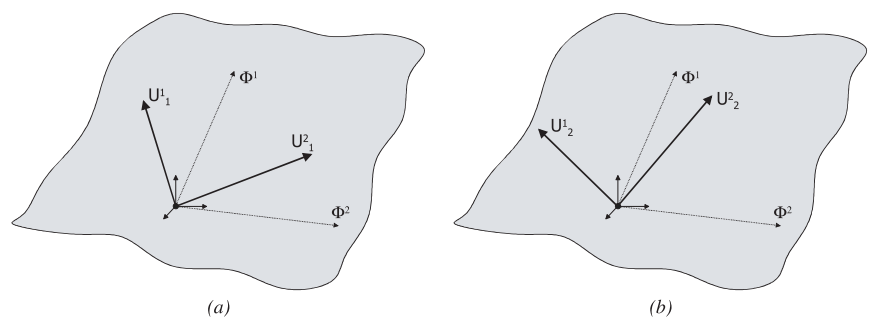

Fig. 1. Composantes principales extraites des observations, (a) jeu de données $\mathrm{n}^{\circ} 1$, (b) jeu de données $\mathrm{n}^{\circ} 2$.

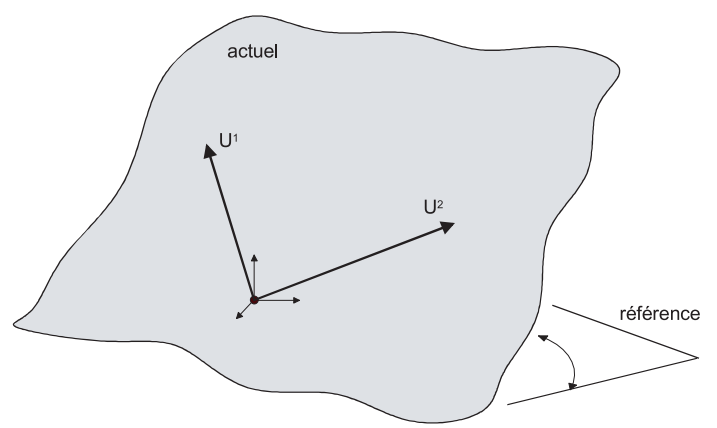

Fig. 2. Rotation de l'hyperplan principal, due à la présence d'un endommagement structural.

Bien que les composantes principales $\mathbf{U}^{1}$ et $\mathbf{U}^{2}$ ne soient pas confondues avec les vecteurs propres $\boldsymbol{\Phi}^{1}$ et $\boldsymbol{\Phi}^{2}$, les plans formés par ces deux paires de vecteurs sont confondus. On notera que les composantes principales sont orthogonales entre-elles, tandis que les vecteurs propres structuraux sont orthogonaux dans la métrique de la matrice de masse $\mathbf{M}$. Au contraire des vecteurs propres structuraux, les composantes principales ne sont pas invariantes, seul l'hyperplan formé par les composantes principales est, quant à lui invariant. Ainsi, les composantes principales, associées à deux jeux d'observations pour deux intervalles de temps distincts, peuvent être différents mais restent coplanaires à l'hyperplan des vecteurs propres (Fig. 1b).

Mathématiquement, ceci signifie que l'hyperplan «principal » est invariant, même si les composantes principales sont dépendantes de l'excitation structurale. Par contre, l'hyperplan principal dépend des caractéristiques structurales et, a fortiori, de la présence d'un endommagement. La détection de la présence d'un endommagement structural peut alors s'effectuer en caractérisant la cohérence spatiale entre l'hyperplan de référence (correspondant à la structure saine) et l'hyperplan actuel (Fig. 2).

Pour caractériser la cohérence spatiale entre deux hyperplans, on peut utiliser le concept d'angles entre sousespaces ou mesurer l'erreur de reconstruction des données expérimentales sur l'hyperplan de référence.

La localisation du dommage à l'aide de cette méthode a également été proposée dans la référence [8]. L'idée de base consiste à identifier les capteurs présentant une erreur de reconstruction maximum ou les plus sensibles à la variation de l'angle entre les hyperplans successifs.

\subsection{Angles entre sous espaces}

Le concept d'angles entre sous-espaces, introduit par Golub et Van Loan [11], permet de quantifier la cohérence spatiale existant entre deux jeux de mesures issus de l'observation d'un système.

Soient deux sous-espaces (ou hyperplans) formés par les colonnes (supposées linéairement indépendantes) de deux matrices $\mathbf{A} \in \Re^{\ell \times p}$ et $\mathbf{B} \in \Re^{\ell \times q}(p \geq q)$. La procédure d'estimation d'angles entre sous-espaces débute par une factorisation $Q R$ des matrices $\mathbf{A}$ et $\mathbf{B}$ :

$$
\begin{array}{ll}
\mathbf{A}=\mathbf{Q}_{A} \mathbf{R}_{A} & \mathbf{Q}_{A} \in \Re^{\ell \times p} \\
\mathbf{B}=\mathbf{Q}_{B} \mathbf{R}_{B} & \mathbf{Q}_{B} \in \Re^{\ell \times q}
\end{array}
$$

Les matrices $\mathbf{Q}_{A}$ et $\mathbf{Q}_{B}$ définissent les bases orthonormées des sous-espaces définis par les colonnes de $\mathbf{A}$ et $\mathbf{B}$. Les angles $\theta_{i}$ formés par les sous-espaces $\mathbf{A}$ et $\mathbf{B}$ sont identifiés à partir des valeurs singulières associées au produit $\mathbf{Q}_{A}^{T} \mathbf{Q}_{B}$ :

$$
\begin{aligned}
\mathrm{Q}_{A}^{T} \mathrm{Q}_{B} & =\mathrm{U}_{A B} \boldsymbol{\Sigma}_{A B} \mathbf{V}_{A B}^{T} \\
\boldsymbol{\Sigma}_{A B} & =\operatorname{diag}\left(\cos \left(\theta_{i}\right)\right) \quad i=\mathbf{1}, \ldots, q
\end{aligned}
$$

La plus petite valeur singulière est donc associée au plus grand angle caractérisant la différence géométrique entre les deux hyperplans.

\subsection{Erreurs de reconstruction des données expérimentales}

La technique d' « erreurs de reconstruction des données expérimentales » est plus connue par sa terminologie anglophone «Novelty Analysis ». Cette technique quantifie le résidu produit par la comparaison entre des réponses structurales mesurées et leurs prédictions correspondantes.

L'erreur de reconstruction peut être estimée à partir de la relation :

$$
\mathbf{E}=\mathbf{Y}-\hat{\mathbf{Y}}=\mathbf{Y}-\mathbf{U}_{1} \mathbf{U}_{1}^{T} \mathbf{Y}
$$

La norme de l'erreur au sens d'Euclide ou de Mahalanobis est calculée à partir du Novelty Index $(N I)$, pour l'état de référence et l'état actuel respectivement. L'accroissement du niveau de l'indicateur $N I$ révèle la présence d'un endommagement structural.

\subsection{Amélioration de la technique PCA}

Le diagnostic de défauts avec la méthode PCA présente les deux limitations suivantes :

a) le nombre de capteurs doit être sensiblement plus grand que le nombre de modes participant à la réponse; 

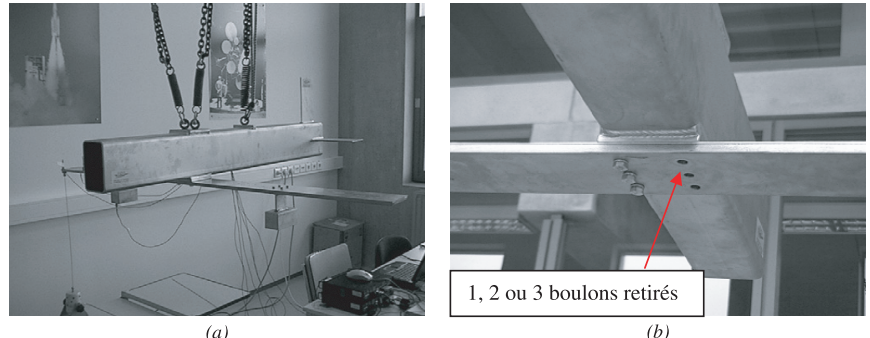

Fig. 3. Vue d'ensemble de l'avion (a), détails du dommage (b).

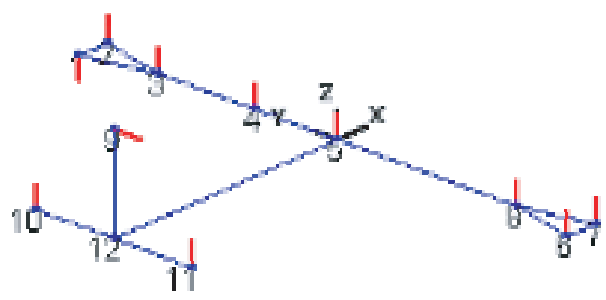

Fig. 4. Répartition des 11 capteurs.

b) on a vu que l'analyse en composantes principales de la matrice des observations $\mathbf{Y}$ définit un hyperplan confondu avec l'ensemble des modes propres structuraux sans intervenir les valeurs propres. Il peut arriver que la sensibilité des modes propres (et donc des composantes principales) à un endommagement structural soit très réduite.

Une alternative à la méthode ci-dessus consiste à effectuer une analyse en composantes principales sur la matrice de Hankle (5) ou (9), construite à partir des différentes observations. Cette variante proposée est sensible aux variations non seulement sur les modes propres, mais aussi sur les fréquences de résonance et les amortissements modaux associés. Cette solution permet alors de surmonter les deux limitations précédentes.

\section{Exemples d'application}

Les méthodes décrites ci-dessus sont illustrées sur l'exemple d'une maquette d'avion en acier suspendue aux moyens de ressorts métalliques comme montré à la figure $3 \mathrm{a}$. La longueur de l'avion est de 1,2 m et l'envergure de $1,5 \mathrm{~m}$.

Une excitation aléatoire est appliquée sur l'aile gauche par l'intermédiaire d'un excitateur électrodynamique dans la bande de fréquence $0-130 \mathrm{~Hz}$. La structure a été instrumentée à l'aide de 11 accéléromètres distribués sur les ailes et la queue (Fig. 4). Trois niveaux d'endommagement ont été créés en retirant successivement un, deux puis trois boulons de fixation de l'aile droite, comme illustré à la figure $3 \mathrm{~b}$.

La méthode «data-driven $S S I$ » a été utilisée pour identifier les paramètres modaux de la structure pour les différents scénarios d'endommagement (niveau 1 à 3), le niveau 0 correspondant à la structure saine. La figure 5 donne un exemple de détection d'endommagement dans le
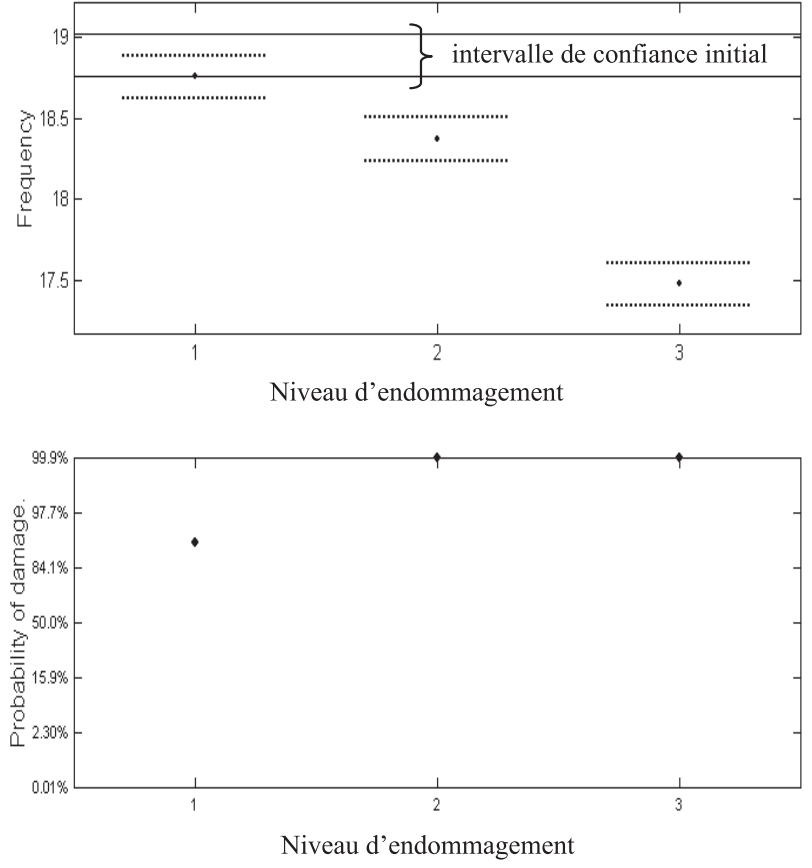

Fig. 5. Détection de l'endommagement à partir de l'identification du premier mode.

cas où seul le mode 1 est considéré. Ces résultats montrent que le niveau 1 (correspondant à un endommagement faible) n'est pas clairement détecté tandis que les niveaux 2 et 3 sont clairement identifiés. Une analyse statistique peut fournir aussi une estimation sur la probabilité d'endommagement, voir la figure 5 (bas).

À titre d'exemple, le diagramme de l'indicateur NI, obtenu avec l'observateur de Kalman, est donné à la figure 6 pour le niveau 2 d'endommagement. La partie gauche du diagramme (8000 points d'échantillonnage) sert de référence et la partie droite correspond aux nouvelles observations. Les statistiques (en \%), et le rapport des valeurs moyennes de l'indicateur $N I$ (marqué $e_{2} / e_{0}$ ) démontrent sans contestation possible l'existence d'un endommagement.

L'ensemble des résultats obtenus par la méthode de reconstruction du modèle de Kalman est repris au tableau 1. Ces résultats sont tout à fait comparables à ceux obtenus par la méthode SSI. La troisième colonne du tableau est relative à un test de « fausse alarme » : elle correspond à un ensemble de données relevées sur la structure saine mais à un niveau d'excitation d'amplitude plus élevée (multipliée par 1,5). On constate que les indicateurs d'endommagement restent faibles malgré les amplitudes d'excitation très différentes.

Le tableau 2 reprend les résultats obtenus avec la méthode basée sur l'analyse modifiée en composantes principales. Les résultats donnés sont en bon accord avec les analyses précédentes.

La détection et la localisation du dommage avec la méthode PCA modifiée peuvent également être réalisées en calculant l'angle entre les hyperplans. Les résultats 
Tableau 1. Détection d'endommagement par le modèle de Kalman.

\begin{tabular}{cccccc}
\hline & $\begin{array}{c}\text { État 0 } \\
\text { (référence) }\end{array}$ & $\begin{array}{c}\text { État 0 } \\
\text { (fausse alarme) }\end{array}$ & État 1 & État 2 & État 3 \\
\hline Dépassements & $1,1 \%$ & $2,7 \%$ & $14,9 \%$ & $94,3 \%$ & $97,9 \%$ \\
\hline $\bar{N} \bar{I}_{d} / \bar{N} \bar{I}_{r}$ & 1 & 1,14 & 1,68 & 6,15 & 6,67 \\
\hline
\end{tabular}

Tableau 2. Détection d'endommagement à partir de la méthode PCA.

\begin{tabular}{cccccc}
\hline & $\begin{array}{c}\text { État 0 } \\
\text { (référence) }\end{array}$ & $\begin{array}{c}\text { État 0 } \\
\text { (fausse alarme) }\end{array}$ & État 1 & État 2 & État 3 \\
\hline Dépassements & $0,1 \%$ & $1,1 \%$ & $11,6 \%$ & $73,3 \%$ & $60,2 \%$ \\
\hline $\bar{N} \bar{I}_{d} / \bar{N} \bar{I}_{r}$ & 1 & 1,05 & 1,19 & 1,69 & 1,61 \\
\hline
\end{tabular}

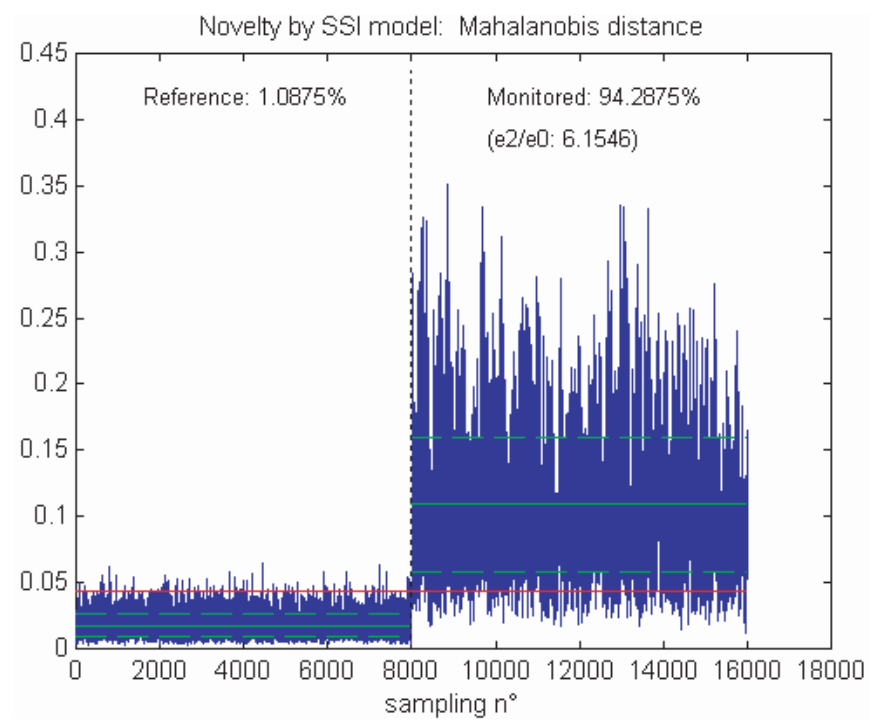

Fig. 6. Détection d'endommagement par analyse statistique basée sur le modèle de Kalman (niveau 2 d'endommagement).

sont présentés à la figure 7. L'endommagement correspond à un angle élevé entre les hyperplans de référence et actuel. Lorsque les données correspondant aux capteurs 6-8 (capteurs situés sur l'aile droite) sont retirées, l'angle décroît. Ceci indique que l'endommagement se situe dans l'aile droite de la structure d'avion.

\section{Conclusions}

Cet article résume plusieurs techniques destinées à la surveillance et au diagnostic de structures (SHM). On notera que les méthodes proposées ne requièrent pas la mesure de l'excitation structurale.

La technique SSI a été employée pour fournir une identification précise des paramètres modaux et estimer un observateur optimum au sens de Kalman. On a également utilisé une analyse en composantes principales afin de traiter du problème de la détection de défaut. Une amélioration de la méthode a également été proposée.

La grande simplicité d'implémentation, ainsi que la faible charge de calcul associées à la méthode PCA

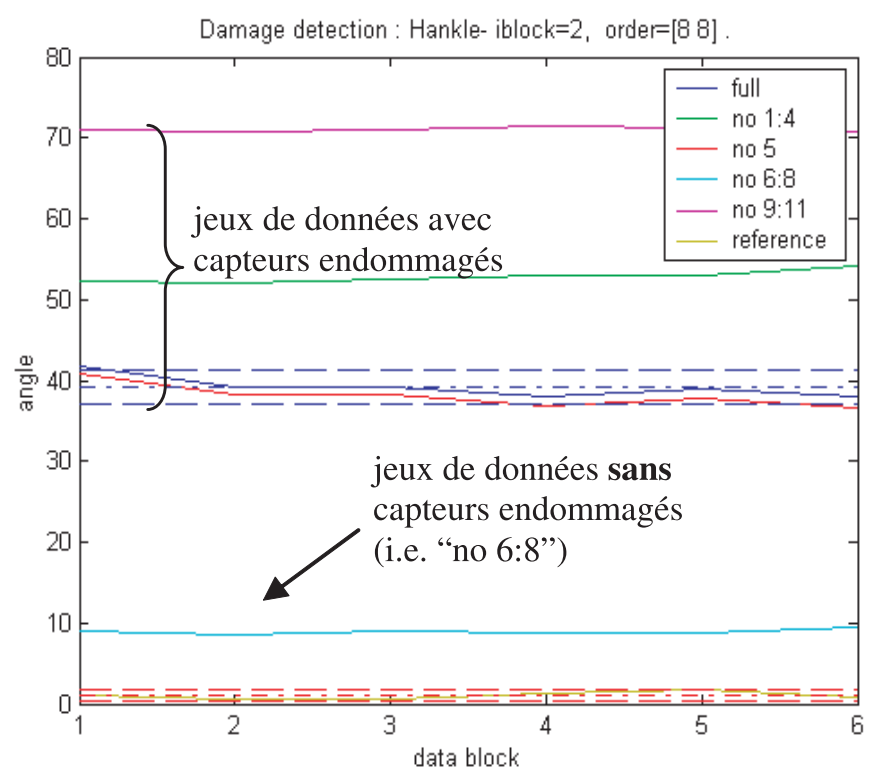

Fig. 7. Résultats de la localisation par PCA modifiée.

devraient pouvoir permettre une utilisation de cette technique pour la détection d'endommagement en temps quasi-réel.

Remerciements. Ce travail a été réalisé dans le cadre d'une convention de recherche $n^{\circ} 13419$ financée par la région wallonne de Belgique.

\section{Références}

[1] M.I. Friswell, J.E. Mottershead, Finite element model updating in structural dynamics, The Netherlands : Kluwer Academic Publishers, 1995

[2] S.W. Doebling, C.R. Farrar, M.B. Prime, D.W. Shevitz, Los Almos National Laboratory report LA-13070-MS, 1996

[3] P. Van Overschee, B. De Moor, Subspace identification for linear systems : theory implementation - applications. Dordrecht, Netherlands : Kluwer Academic Publishers, 1996 
[4] B. Peeters, System Identification and Damage Detection in Civil Engineering, Ph.D., Dissertation, Katholieke Universiteit Leuven, Belgium, 2000

[5] H. Sohn, K. Worden, C.F. Farrar, Novelty detection under changing environmental conditions, SPIE's 8th Annual Int. Symposium on Smart structures and materials, Newport Beach, CA, 2001

[6] M.I. Friswell, D.J. Inman, Sensor validation for SMART structures, IMAC XVIII, San Antonio (2000) 483-489

[7] A.M. Yan, P. De Boe, J.C. Golinval, Structural damage diagnosis by Kalman model based on stochastic subspace identification, Int. J. Struct. Health Monitoring 3 (2004) 103-119
[8] P. De Boe, J.-C. Golinval, Principal component analysis of piezo-sensor array for damage localization, Int. J. Struct. Health Monitoring 2 (2003) 137-144

[9] A.K. Pandey, M. Biswas, Damage detection in structures using changes in flexibility, J. Sound and Vibration 169 (1994) 3-17

[10] V. Lenaerts, G. Kershen, J.-C. Golinval, Proper Orthogonal Decomposition for Model Updating of NonLinear Mechanical Systems, Mechanical Systems and Signal Processing 15(1) (2001) 31-43

[11] G.H. Golub, C.F. Van Loan. Matrix Computations, Baltimore, The Johns Hopkins University Press, 1996 